Pacific Journal of Mathematics

ON THE HOMOMORPHIC AND ISOMORPHIC EMBEDDINGS 


\title{
ON THE HOMOMORPHIC AND ISOMORPHIC EMBEDDINGS OF A SEMIFLOW INTO A RADIAL FLOW
}

\author{
Michael Edelstein
}

It is the main purpose of this paper to prove the following two theorems.

Theorem I. (Isomorphism) Let $\left(X, \boldsymbol{R}^{+}, f\right)$ be a semiflow on a separable metric space $(X, d)$, having the properties:

(i) there is an $\omega \in X$ such that, for each neighborhood $U$ of $\omega$, there is a $T \in \boldsymbol{R}^{+}$with $f[X, t] \subset U$ for all $t \geqq T$;

(ii) for each $t \in \boldsymbol{R}^{+}, f(\cdot, t)$ is a homeomorphism of $X$ onto a closed subspace of $X$.

Then $\left(X, \boldsymbol{R}^{+}, f\right)$ is isomorphic to a radial semiflow on a subset of the Hilbert Cube in $l^{2}$.

Theorem II. (Homomorphism) If $\left(X, \boldsymbol{R}^{+}, f\right)$ satisfies the hypotheses of Theorem $I$, with $(i)$ replaced by

$\left(\mathbf{i}^{\prime}\right) \quad \cap\{f[X, t]: t \geqq 0\}=\{\omega\}$ for some $\omega \in X$, then $\left\{X, \boldsymbol{R}^{+}, f\right)$ is homomorphic to a radial semiflow on a subset of the Hilbert Cube $C$ and the subsemiflow induced on $X /\{\omega\}$ is isomorphic to a radial semiflow in $C$.

1. Introduction. Let $X$ be a nonempty subset of a normed linear space and suppose that, with $0<\lambda<1$,

$$
f(x, t)=\lambda^{t} x \quad((x, t) \in X \times R) .
$$

The triple $(X, R, f)$, with $f$ as above, determines a dynamical system or a flow (cf. [4], [5]) on $X$ such that the semitrajectory of each $x \neq 0$ is a line segment joining $x$ with the origin 0 . The terms "a radial flow" or "a radial dynamical system" seem appropriate. Similarly, with $\boldsymbol{R}$ replaced by $\boldsymbol{R}^{+}$, the nonnegative reals, we refer to $\left(X, \boldsymbol{R}^{+}, f\right)$ as a radial semiflow, or a radial semidynamical system. By a homomorphic (isomorphic) embedding of $(X, \boldsymbol{R}, f)$ into $(Y, \boldsymbol{R}, g)$ we understand a one-one continuous mapping (a homeomorphism) $h$ of $X$ into $Y$ such that

$$
h(f(x, t))=g(h(x), t)((x, t) \in X \times \boldsymbol{R}) .
$$

A similar definition applies to semiflows. Thus, to say that $\left(X, \boldsymbol{R}^{+}\right.$, $f$ ) is isomorphic to a radial semiflow means that a homeomorphism $h$ of $X$ into a normed linear space exists such that

$$
h(f(x, t))=\lambda^{t} h(x)\left((x, t) \in X \times \boldsymbol{R}^{+}\right) .
$$

In a recent paper L. Janos [3] proved that a semiflow $\left(X, \boldsymbol{R}^{+}, f\right)$ 
on a compact metric space $X$ is isomorphic to a radial semiflow on a subset $X$ of the Hilbert Cube in $l_{2}$ if the transition map $f$ is oneone and $\cap\{f[X, t]: t \geqq 0\}$ is a singleton.

It is the main purpose of this paper to establish the extensions of the above result to the setting of semiflows on a separable metric space which were stated in the opening paragraph.

As can be readily verified, conditions (i) and (i') are equivalent if $X$ is compact metric. Since (ii) is automatically satisfied for a one-one continuous transition map on a compact metric space, the above mentioned result of Janos follows as a corollary. On the other hand it is quite easy to show that the conclusion of Theorem I cannot be obtained with (i) replaced by (i').

An analog for discrete semiflows on a compact metric space was used by Janos [3] in the proof of his result. While such an analog was available before (cf. [1], [2]), the corresponding one for separable metric spaces given here (Theorems 2 and 3) is new and of independent interest. Also of some interest is the result asserting that the property of radiality is passed on to a flow by the corresponding property of its discrete subflows (Theorem 1). This result parallels the "Embedding Lemma" of [3].

\section{Inheritance of radiality.}

THEOREM 1. Let $(X, R, f)$ be a dynamical system on a nonempty subset $X$ of $l_{2}$ with the property that for some $\lambda, 0<\lambda<1$, all nonnegative integers $n$ and all $(x, t) \in X \times \boldsymbol{R}$,

$$
f(x, t+n)=\lambda^{n} f(x, t) .
$$

Then $(X, \boldsymbol{R}, f)$ is isomorphic to a radial flow in $l_{2}$.

Proof. Applying an idea of M. Bebutov (cf., e.g., [4], p. 333), we consider the integral

$$
\int_{0}^{\infty}\|f(x, t)\| d t
$$

This improper integral converges since

$$
\sum_{m=0}^{\infty} \int_{m}^{m+1}\|f(x, t)\| d t=\sum_{m=0}^{\infty} \int_{0}^{1}\|f(x, t+m)\| d t ;
$$

by (3) then

$$
\int_{0}^{\infty}\|f(x, t)\| d t=\frac{1}{1-\lambda} \int_{0}^{1}\|f(x, t)\| d t
$$


proving convergence. Thus $s_{x}(T)=\int_{T}^{\infty}\|f(x, t)\| d t$ defines a function from $\boldsymbol{R}$ to $\boldsymbol{R}^{+}$.

Clearly, this function is continuous and decreasing for $x$ distinct from the origin 0 . Its range for all $x \neq 0$ contains $\{1\}$ as it consists of all positive reals. [Indeed $\lim _{T \rightarrow \infty} s_{x}(T)=0$ by convergence of the integral in (4) and, on the other hand

$$
s_{x}(-n)>\int_{-n}^{0}\|f(x, t)\| d t \geqq \lambda^{-n} \int_{0}^{1}\|f(x, t)\| d t ;
$$

so that $s_{x}(T) \rightarrow \infty$ as $T \rightarrow-\infty$.]

To each $x \in X \backslash\{0\}$ there corresponds a unique $t_{x}$ such that $s_{x}\left(t_{x}\right)=$ 1. The correspondence $x \rightarrow t_{x}$ which arises in this manner is clearly one-one on trajectories lying in $X \backslash\{0\}$. Also, if $y=f(x, t)$ then, as can be readily seen

$$
t_{y}=t_{x}-t
$$

To prove that $t_{x}$ is continuous in $x-a$ fact needed in the sequellet $\left\{x_{n}\right\}$ be a sequence in $X \backslash\{0\}$ with $x_{n} \rightarrow x \neq 0$. Then, we claim,

$$
\lim _{n \rightarrow \infty} \int_{t_{x}}^{\infty}\left\|f\left(x_{n}, t\right)\right\| d t=1
$$

Indeed,

$$
\begin{aligned}
\int_{t_{x}}^{\infty}\left\|f\left(x_{n}, t\right)\right\| d t & =\int_{t_{x}}^{0}\left\|f\left(x_{n}, t\right)\right\| d t+\sum_{m=0}^{\infty} \int_{m}^{m+1}\left\|f\left(x_{n}, t\right)\right\| d t \\
& =\int_{t_{x}}^{0}\left\|f\left(x_{n}, t\right)\right\| d t+\frac{1}{1-\lambda} \int_{0}^{1}\left\|f\left(x_{n}, t\right)\right\| d t .
\end{aligned}
$$

As $f\left(x_{n}, t\right) \rightarrow f(x, t)$ uniformly on compact intervals ([4], p. 327), we obtain in the limit, as $n \rightarrow \infty$,

$$
\int_{t_{x}}^{\infty}\|f(x, t)\| d t=1
$$

Thus

$$
\int_{t_{x_{n}}}^{t_{x}}\left\|f\left(x_{n}, t\right)\right\| d t=1-\int_{t_{x}}^{\infty}\left\|f\left(x_{n}, t\right)\right\| d t \longrightarrow 0 \text { as } n \longrightarrow \infty .
$$

However this is only possible if $t_{x_{n}} \rightarrow t_{x}$.

Let $S$ be the set of all $x$ in $X$ such that $t_{x}=0$; i.e.,

$$
S=\left\{x \in X: \int_{0}^{\infty}\|f(x, t)\| d t=1\right\} .
$$

In view of the preceding discussion $S$ is a closed subset of $X \backslash\{0\}$ 
and each trajectory, lying in that subset, meets $S$ at exactly one point. (In the usual terminology $S$ is a section in $X \backslash\{0\}$.)

Let $\bar{\sigma}: S \rightarrow \sum$ be a homeomorphism onto a subset of $H \cap C$ where $H$ is the hyperplane $\left\{x \in l_{2}:\left\langle x, e_{1}\right\rangle=1\right\}$, and $C$ denotes the Hilbert Cube in $l_{2}$. [That such a homeomorphism exists follows from the facts that $\left(x_{1}, x_{2}, \cdots\right) \leftrightarrow\left(1, x_{1}, x_{2}, \cdots\right)$ sets up an isometry in $l_{2}$ and that any separable metric space is homeomorphic with a subset of the Hilbert Cube.] The desired isomorphism is now defined as the mapping $\sigma$ of $X$ into $\boldsymbol{R} \sum$ obtained by setting

$$
\sigma(x)=\lambda^{-t_{x}} \bar{\sigma}\left(f\left(x, t_{x}\right)\right)(x \neq 0)
$$

$$
\text { and if } 0 \in X, \sigma(0)=0 \text {. }
$$

On $X \backslash\{0\} \sigma$ is continuous as the composition of $t_{x}, f$ and $\bar{\sigma}$. If $r>$ 0 and $y \in r \Sigma$ then $y=\lambda^{t} \bar{\sigma}(x) \Leftrightarrow \bar{\sigma}^{-1}\left(\lambda^{-t} y\right)=x$ for some $t \in \boldsymbol{R}$; hence (the existence and) continuity of the inverse. To prove continuity at 0 of both $\sigma$ and $\sigma^{-1}$ it suffices, in view of the definition (9), to show that a sequence $\left\{x_{n}\right\}$ in $X \backslash\{0\}$ converges to 0 if, and only if, $t_{x_{n}} \rightarrow-\infty$.

Suppose, first, that $t_{x_{n}} \rightarrow-\infty$. If $\left\{x_{n}\right\}$ fails to converge to 0 then we may assume that $\left\|x_{n}\right\| \geqq \varepsilon$ for some $\varepsilon>0$. As a result,

$$
M=\inf \left\{\left\|f\left(x_{n}, t\right)\right\|: n=1,2, \cdots ; 0 \leqq t \leqq 1\right\}>0 .
$$

[Otherwise a sequence $\left\{t_{i}\right\}$ in $[0,1]$ and a subsequence $\left\{x_{n_{i}}\right\}$ would have to exist such that $t_{i} \rightarrow t^{*} \in[0,1]$ and $f\left(x_{n_{i}}, t_{i}\right) \rightarrow 0$. But then $f\left(x_{n_{i}}, t^{*}\right)=\left(f\left(x_{n_{i}}, t^{*}-t_{i}+t_{i}\right)\right)=f\left(f\left(x_{n_{i}}, t_{i}\right), \quad t_{i}^{*}-t_{i}\right) \rightarrow 0$, implying $x_{n_{i}} \rightarrow 0$.] Hence by (8), (cf. also (5)),

$$
\begin{gathered}
1=\int_{t_{x_{n}}}^{\infty}\left\|f\left(x_{n}, t\right)\right\| d t=\int_{t_{x_{n}}}^{-m}\left\|f\left(x_{n}, t\right)\right\| d t+\frac{\lambda^{-m}}{1-\lambda} \int_{0}^{1}\left\|f\left(x_{n}, t\right)\right\| d t \\
\geqq(1-\lambda)^{-1} \lambda^{-m} M, \text { if }-m-1 \leqq t_{x_{n}} \leqq-m .
\end{gathered}
$$

However, for sufficiently large $n, \lambda^{-m} M>1-\lambda$, leading to a contradiction.

Next, let $x_{n} \rightarrow 0$ and assume, for a contradiction, that $t_{x_{n}} \rightarrow-\infty$. Then we may assume that $t_{x_{n}} \geqq-m$, where $m$ is a fixed positive integer. Hence,

$$
\begin{array}{r}
1=\int_{t_{x_{n}}}^{\infty}\left\|f\left(x_{n}, t\right)\right\| d t \leqq \int_{-m}^{\infty}\left\|f\left(x_{n}, t\right)\right\| d t \\
=(1-\lambda)^{-1} \lambda^{-m} \int_{0}^{1}\left\|f\left(x_{n}, t\right)\right\| d t .
\end{array}
$$

However, as already observed, $x_{n} \rightarrow 0 \Rightarrow \int_{0}^{1}\left\|f\left(x_{n}, t\right)\right\| d t \rightarrow 0$ which is impossible. 
Finally, with $f(x, t)=y$, and by a repeated application of (6),

$$
\begin{aligned}
\sigma(f(x, t)) & =\lambda^{-t_{y}} \bar{\sigma}\left(f\left(y, t_{y}\right)\right)=\lambda^{-t_{x}+t} \bar{\sigma}\left(f\left(x, t+t_{y}\right)\right) \\
& =\lambda^{t} \lambda^{-t_{x}} \bar{\sigma}\left(f\left(x, t_{x}\right)\right)=\lambda^{t} \sigma(x)
\end{aligned}
$$

showing that $(X, \boldsymbol{R}, f)$ is isomorphic with the radial flow $(\boldsymbol{R} \Sigma, \boldsymbol{R}, g)$, where $g(x, t)=\lambda^{t} x$.

3. Discrete semiflows. In analogy with the definition of a (continuous) radial semiflow we define a discrete radial semiflow $\left(X, N^{+}, f\right)$ on a nonempty set $X$ in a normed linear space as one in which

$$
f(x, n)=\lambda^{n} x
$$

for some $\lambda, 0<\lambda<1$, and all $(x, n) \in X \times N^{+}$.

A continuous mapping $f$ of a metric space $M$ into itself determines a discrete semiflow $\left(M, N^{+}, \bar{f}\right)$ by setting $\bar{f}(x, n)=f^{n}(x)$. Hence a homeomorphism (a one-one continuous mapping) $h: M \rightarrow l_{2}$ satisfying

$$
h(f(x))=\lambda h(x)
$$

with $0<\lambda<1$, determines an isomorphic (a homomorphic) embedding into a radial semiflow in $l_{2}$; namely one for which $h(\bar{f}(x, n))=$ $\lambda^{n} h(x)$.

For a mapping $h$ to satisfy (*) it is clearly necessary and sufficient that the "coordinate functions" $\psi_{n}(x)=\left\langle h(x), e_{n}\right\rangle,(n=1,2, \cdots)$, where $e_{n}$ is a member of the standard orthonormal basis, do likewise; i.e.,

$$
\psi_{n}(f(x))=\lambda \psi_{n}(x)
$$

Further, for $h$ to be one-one it is necessary and sufficient that the family $\left\{\psi_{n}\right\}$ distinguishes points; i.e., if $x^{\prime}, x^{\prime \prime}$ are distinct members of $X$ then $\psi_{n}\left(x^{\prime}\right) \neq \psi_{n}\left(x^{\prime \prime}\right)$ for some $n \in N^{+}$.

By means of such a family of functions it was shown in [1] that a homeomorphism $h: X \rightarrow l_{2}$ exists satisfying (*) if $X$ is compact metric, $f: X \rightarrow X$ is one-one continuous, and

$$
\cap\left\{f^{n}[X]: n=1,2, \cdots\right\}=\{\omega\}
$$

where $\omega \in X$.

If compactness is removed from the hypotheses of the above result then, as simple examples show, the conclusions may no longer be true even if $f$ is a homeomorphism (onto $f[X]$ ). This is due to the fact that in general $h$ may fail to have a continuous inverse 
at some points of $h[X]$. Furthermore, the construction of a homeomorphism $h$ such that $h^{-1}$ is continuous at some points, e.g., on $h[X] \backslash\{0\}$, as in the next theorem, seems inadequate. Hence the need for the more refined construction which we produce in the proofs of the following theorems.

\subsection{Homomorphisms.}

THEOREM 2. Let $X$ be a separable metric space and $f$ a homeomorphism of $X$, onto a closed proper subset of $X$, satisfying (11). Then there is a one-one continuous mapping $h$ of $X$ into the Hilbert Cube $C$ in $l_{2}$ such that $h^{-1}$ is continuous on $h[X] \backslash\{0\}$ and (*) holds.

Proof. Let $\mathscr{B}$ be a countable base for the topology of $X \backslash f[X]$ and let $\left\{\left(U_{n}, V_{n}\right): n=1,2, \cdots\right\}$ be an enumeration of those pairs $(U, V) \in \mathscr{B} \times \mathscr{B}$ for which $\bar{U} \subset V$. Set

$$
\begin{aligned}
B_{n}^{(k)}=U\left\{B(x, r(x)): x \in f^{k}\left[V_{n}\right]\right\}, \\
\quad(n=1,2, \cdots ; k=0,1, \cdots),
\end{aligned}
$$

where $B(x, r(x))$ denotes the open ball about $x$, of radius $r(x)=$ $2^{-1} d\left(x, f^{k}[X] \backslash f^{k}\left[V_{n}\right]\right.$ ); (with this choice of $r(x)$ we have $B_{n}^{(i)} \cap B_{n}^{(j)}=$ $\varnothing$ for $i \neq j$ ).

For $k=0,1, \cdots$ let $\alpha_{2 n-1}^{(k)}$ be a continuous mapping into $[0,1]$ such that

$$
\begin{aligned}
& \alpha_{2 n-1}^{(k)}\left[X \backslash \cup\left\{B_{n}^{(i)}: i=0,1, \cdots, k\right\}\right]=0 \\
& \alpha_{2 n-1}^{(k)}\left[U_{n} \cup f\left[U_{n}\right] \cup \cdots \cup f^{k}\left[U_{n}\right]\right]=1
\end{aligned}
$$

Since $B_{n}^{(i)}$ is disjoint from $f^{k}[X]$ for $i \leqq k$ we have

$$
\alpha_{2 n-1}^{(k)} f^{k+1}=0 \text {. }
$$

To define $\alpha_{2 m(2 n-1)}^{(k)}$ for integers $m \neq 0$ pick, for $m>0$, an arbitrary continuous extension of $\bar{\alpha}_{2^{m-1}\left(n_{2-1}\right)}^{(k)}$, where

$$
\bar{\alpha}_{2^{m-1}(2 n-1)}^{(k)}=\left\{\begin{array}{l}
\alpha_{2^{m-1}(2 n-1)}^{(k)} f^{-1} \text { on } f[X] \\
1 \text { on } U_{n}
\end{array}\right.
$$

and, for other subscripts

$$
\alpha_{2^{-m}(2 n-1)}^{(k)}=\alpha_{2^{-m+1}(2 n-1)}^{(k)} f .
$$

Thus

$$
\alpha_{2^{m}(2 n-1)}^{(k)} f=\alpha_{2^{m-1}(2 n-1)}^{(k)}(m=0, \pm 1, \cdots ; n=1,2, \cdots)
$$


A countable family of coordinate functions $\psi_{n}^{(k)}: X \rightarrow[0,1]$ is now obtained by setting

$$
\psi_{n}^{(k)}=(1-\lambda) \sum_{m=-k}^{\infty} \lambda^{m+k} \alpha_{2^{m}(2 n-1)}^{(k)} .
$$

By (12), and the fact that $\alpha_{2-k(2 n-1)}^{(k)} f=\alpha_{2 n-1}^{(k)} f^{k+1}=0$, we have

$$
\begin{aligned}
\psi_{n}^{(k)} f & =(1-\lambda) \sum_{m=-k}^{\infty} \lambda^{m+k} \alpha_{2^{(k)}(2 n-1)}^{(k)} f \\
& =(1-\lambda) \sum_{m=-k+1} \lambda^{m^{*}+k} \alpha_{2^{m}(2 n-1)}^{(k)} f \\
& =\lambda(1-\lambda) \sum_{m=-k+1} \lambda^{m+k-1} \alpha_{2^{m-1}(2 n-1)}^{(k)}=\lambda \psi_{n}^{(k)},
\end{aligned}
$$

showing that (10) holds for all coordinate functions. To define $h$ : $X \rightarrow C \subset l_{2}$ let $\left\{\psi_{j}: j=1,2, \cdots\right\}$ be an enumeration of $\left\{\psi_{n}^{(k)}\right\}$ and set

$$
h(x)=\left(\frac{\psi_{1}(x)}{1}, \frac{\psi_{2}(x)}{2}, \cdots, \frac{\psi_{j}(x)}{j}, \cdots\right) .
$$

A standard argument, omitted here, shows that $h$ is continuous. To prove that $h$ is one-one, let $x_{1}, x_{2}$ be distinct points of $X$ and suppose that $x_{1}=f^{k_{1}}\left(y_{1}\right), x_{2}=f^{k_{2}}\left(y_{2}\right)$ with $k_{1} \leqq k_{2}$ and $y_{1} \in X \backslash f[X]$. Choose $n$ such that $\alpha_{2 n-1}^{(0)}\left(y_{1}\right)=1$ and $\alpha_{2 n-1}^{(0)}\left(y_{2}\right)=0$. Then

$$
\psi_{n}^{(0)}\left(y_{1}\right)=(1-\lambda) \sum_{m=0}^{\infty} \lambda^{m} \alpha_{2 m(2 n-1)}^{(0)}\left(y_{1}\right)=1 \Longrightarrow \psi_{n}^{(0)}\left(x_{1}\right)=\lambda^{k_{1}}
$$

while

$$
\psi_{n}^{(1)}\left(y_{2}\right)=(1-\lambda) \sum_{m=1}^{\infty} \lambda^{m} \alpha_{2^{m}(2 n-1)}^{(0)}\left(y_{2}\right) \leqq \lambda \Longrightarrow \psi_{n}^{(0)}\left(x_{n}\right) \leqq \lambda^{k_{2}+1}
$$

Hence $\psi_{n}^{(0)}\left(x_{2}\right) \leqq \lambda \psi_{n}^{(0)}\left(x_{1}\right)$ and $h\left(x_{1}\right) \neq h\left(x_{2}\right)$.

To prove continuity of $h^{-1}$ at $h(x), x \neq 0$, let $\left\{h\left(x_{n}\right): n=1,2, \cdots\right\}$ be a sequence in $h[X] \backslash\{0\}$ converging to $h(x)$ and suppose that $x=$ $f^{k}(y)$ for some $k \geqq 0$ and $y \in X \backslash f[X]$. Suppose $x_{n} \rightarrow x$. Quite clearly a subsequence $\left\{x_{n_{i}}\right\}$ and an $\varepsilon>0$ must then exist such that $d\left(x_{n_{i}}\right.$, $\left.f^{j}(y)\right) \geqq \varepsilon$ for all $i=1,2, \cdots$ and $j=0,1, \cdots, k$. [If not, then a subsequence $\left\{x_{n_{i}}\right\}$ exists such that $x_{n_{i}} \rightarrow x$ but $x_{n_{i}} \rightarrow f^{j}(y)$ for some $j \neq k$; but then $h\left(x_{n_{i}}\right) \rightarrow h\left(f^{j}(y)\right)=\lambda^{j-k} h(x) \neq h(x)$.] Let $n$ be such that each $B_{n}^{(j)}$ is contained in the open ball about $f^{j}(y)$, of radius $\varepsilon$ and $y \in V_{n}$. Then

$$
\alpha_{2 n-1}^{(k)}\left(x_{n_{i}}\right)=0 \text { and } \alpha_{2 n-1}^{(k)}\left(f^{j}(y)\right)=1(j=1,2, \cdots, k) .
$$

Hence $\psi_{n}^{(k)}(y)=1 \Rightarrow \psi_{n}^{(k)}(x)=\lambda^{k}$; while 


$$
\begin{aligned}
\psi_{n}^{(k)}\left(x_{n_{i}}\right) & =(1-\lambda) \sum_{m=-k}^{\infty} \lambda^{m+k} \alpha_{2^{m}(2 n-1)}^{(k)}\left(x_{n_{i}}\right) \\
& =(1-\lambda) \lambda^{k} \sum_{m=1}^{\infty} \lambda^{m} \alpha_{2^{m}(2 n-1)}^{(k)}\left(x_{n_{i}}\right) \leqq \lambda^{k+1}=\lambda \psi_{n}^{(k)}(x)
\end{aligned}
$$

Clearly then $\left\|h\left(x_{n_{i}}\right)-h(x)\right\|$ is bounded away from zero, against the assumption that $h\left(x_{n}\right) \rightarrow h(x)$.

3.2. Isomorphism. In the next theorem we have the stronger conclusion that $h$ is a homeomorphism. As in the case of Theorem $I$, where (i) is stronger than ( $\left.i^{\prime}\right)$, it is necessary to strengthen condition (11) by the discrete counterpart of condition (i). Thus we shall assume that the following hypothesis is satisfied.

There exists a $\omega \in X$ such that for every neighborhood $U$ of $\omega$ there is a positive integer $N$ with the property that

$$
f^{n}[X] \subset U
$$

for $n \geqq N$.

(A simple argument shows that in a compact metric space (11) and (13) are equivalent.)

THEOREM 3. Let $X$ be as in Theorem 2 with (11) replaced by (13). Let $0<\lambda<1$. Then a homeomorphism $h$ of $X$ into the Hilbert Cube $C$ in $l_{2}$ exists such that $h(f(x))=\lambda h(x)$.

Proof. Let $B_{n}$ be an open ball about $\omega$, of radius $\varepsilon / n$ with $\varepsilon>0$ such that $B_{1} \neq X$. By (13) there are integers $N_{1}<N_{2}<\cdots$ $<N_{n}<\cdots$ such that

$$
f^{N_{n}}[X] \subset B_{n} .
$$

Let $\alpha_{2 n-1}: X \rightarrow[0,1]$ be a continuous function such that $\alpha_{2 n-1}\left[f^{N_{n}}[X]\right]=$ 0 and $\alpha_{2 n-1}\left[X \backslash B_{n}\right]=1$.

Let $\alpha_{2(2 n-1)}$ be a continuous extension of $\alpha_{2 n-1} f^{-1}$ to the whole of $X$ and, recursively, let $\alpha_{2^{m(2 n-1)}}$ be a continuous extension of $\alpha_{2^{m-1(2 n-1)}} f^{-1}$ to the whole of $X$. Define $\alpha_{2^{-m}(2 n-1)}: X \rightarrow[0,1](m=$ $1,2, \cdots)$ by setting

$$
\alpha_{2-m(2 n-1)}=\alpha_{2-m+1} f .
$$

This defines a family $\left\{\alpha_{2^{m(2 n-1)}}: m=0, \pm 1, \cdots ; n=1,2, \cdots\right\}$ of continuous mappings into $[0,1]$ such that for all integers $m$,

$$
\alpha_{2^{m}(2 n-1)} f=\alpha_{2^{m-1}(2 n-1)}
$$

and 


$$
\alpha_{2^{m(2 n-1)}}=0\left(m \leqq-N_{n}\right)
$$

Let now

$$
\begin{aligned}
\tilde{\Psi}_{n}(x)= & (1-\lambda) \lambda^{N_{n}-1} \sum_{m=-N_{n}+1}^{\infty} \lambda^{m} \alpha_{2^{m}(2 n-1)}(x) \\
& (n=1,2, \cdots ; x \in X) .
\end{aligned}
$$

Then $\widetilde{\psi}_{n}: X \rightarrow[0,1]$ is continuous and by (14) and (15),

$$
\begin{aligned}
\widetilde{\psi}_{n}(f(x)) & =(1-\lambda) \lambda^{N_{n}-1} \sum_{-N_{n}+1}^{\infty} \lambda^{m} \alpha_{2 m(2 n-1)}(f(x)) \\
& =(1-\lambda) \lambda^{N_{n}-1} \sum_{-N_{n}+1}^{\infty} \lambda^{m} \alpha_{2^{m-1}(2 n-1)}(x) \\
& =(1-\lambda) \lambda^{N_{n}} \sum_{-N_{n}+1}^{\infty} \lambda^{m} \alpha_{2^{m}(2 n-1)}(x)=\lambda \widetilde{\psi}_{n}(x)
\end{aligned}
$$

showing that (10) is satisfied.

To define $h: X \rightarrow l_{2}$ let $\left\{\psi_{j}: j=1,2, \cdots\right\}$ be an enumeration of $\left\{\widetilde{\psi}_{n}\right\} \cup\left\{\psi_{n}^{(k)}\right\}$ where $\left\{\psi_{n}^{(k)}\right\}$ is as in the proof of Theorem 2. Set

$$
h(x)=\left(\frac{\psi_{1}(x)}{1}, \frac{\psi_{2}(x)}{2}, \cdots, \frac{\psi_{j}(x)}{j}, \cdots\right) .
$$

The continuity of $h$, the existence of $h^{-1}$ and the continuity of $h^{-1}$ at $h(x) \neq 0$ follows, as in the proof of Theorem 2 , from the relevant properties of $\left\{\psi_{n}^{(k)}\right\}$. To prove continuity of $h^{-1}$ at 0 let $\left\{h\left(x_{n}\right)\right\}$ be a sequence converging to 0 and suppose that $\left\{x_{n}\right\}$ fails to converge to 0 . Then a subsequence $\left\{x_{n_{i}}\right\}$ and a positive integer $n$ exist such that $\left\{x_{n_{i}}\right\}$ is in $X \backslash B_{n}$. Hence $\alpha_{2 n-1}\left(x_{n_{i}}\right)=1 . \quad(i=1,2, \cdots)$, and therefore $\tilde{\psi}_{n}\left(x_{n_{i}}\right) \geqq(1-\lambda) \lambda^{N_{n}-1}$. It follows that $\left\|h\left(x_{n_{i}}\right)\right\|$ is bounded away from zero, against the assumption that $\left\{h\left(x_{n}\right)\right\}$ converges to the origin.

\section{Main results.}

Proof of Theorem I. Fix $\lambda, 0<\lambda<1$, and set $\bar{f}(x)=f(x, 1)$. The mapping $\bar{f}: X \rightarrow X$ satisfies the hypotheses of Theorem 3 . Hence there is a homeomorphism $h$ of $X$ into the Hilbert Cube with $h(\bar{f}(x))=$ $\lambda h(x)$ or, in terms of discrete semiflows,

$$
h(f(x, n))=h\left(\bar{f}^{n}(x)\right)=\lambda^{n}(h(x)),(x \in X) .
$$

Let $Y$ be the set of all $y$ in $l_{2}$ with the property that for some nonnegative integer $n, \lambda^{n} y \in h[X]$, and define $g: Y \times \boldsymbol{R} \rightarrow Y$ by setting

$$
g(y, t)=\lambda^{-m-n} h\left(f\left(h^{-1}\left(\lambda^{n} y\right), t+m\right)\right)
$$


where $m$ and $n$ are the smallest nonnegative integers such that $\lambda^{n} y \in h[X]$ and $t+m \geqq 0$.

From the fact that

$$
f\left(x, t+m+m^{\prime}\right)=f\left(f(x, t+m), m^{\prime}\right)\left(x \in X ; t+m \geqq 0 ; m^{\prime} \geqq 0\right)
$$

and

$$
h\left(f\left(f(x, t+m), m^{\prime}\right)\right)=\lambda^{m^{\prime}} h(f(x, t+m))
$$

it follows that

$$
\lambda^{-m-m^{\prime}} h\left(f\left(x, t+m+m^{\prime}\right)\right)=\lambda^{-m} h(f(x, t+m))
$$

showing that (16) holds for any nonnegative integer $m$ with $m+$ $t \geqq 0$ (and not only for the smallest one with the said property). A similar argument, omitted here, shows that (16) remains true with $n$ replaced by $n^{\prime}$.

To verify the additivity of $g$ let

$$
g\left(y, t+t^{\prime}\right)=\lambda^{-m-m^{\prime}-n} h\left(f\left(h^{-1}\left(\lambda^{n} y\right), t+t^{\prime}+m+m^{\prime}\right)\right)
$$

where $\lambda^{n} y \in h[X], t+m \geqq 0, t^{\prime}+m^{\prime} \geqq 0$. We have

$$
h\left(f\left(h^{-1}\left(\lambda^{n} y\right), t+m\right)\right)=\lambda^{m+n} g(y, t) \in h[X]
$$

and

$$
g\left(g(y, t), t^{\prime}\right)=\lambda^{-m-n-m^{\prime}} h\left(f\left(h^{-1}\left(\lambda^{m+n} g(y, t), t^{\prime}+m^{\prime}\right)\right)\right)
$$

showing that

$$
g\left(y, t+t^{\prime}\right)=g\left(g(y, t), t^{\prime}\right) .
$$

To sum up, $Y$ is a nonempty subset of $l_{2},(Y, R, g)$ is a dynamical system and for $t \in \boldsymbol{R}, k$ a nonnegative integer, we obtain (by choosing positive integers $n, m$ with $\lambda^{n} y \in h[X], t+m \geqq 0$ ) that

$$
\begin{aligned}
g(y, t+k) & =\lambda^{-m-n} h\left(f\left(h^{-1}\left(\lambda^{n} y\right), t+k+m\right)\right) \\
& =\lambda^{k} \lambda^{-m-n} h\left(f\left(h^{-1}\left(\lambda^{n} y^{\prime}, t+m\right)\right)\right)=\lambda^{k} g(y, t)
\end{aligned}
$$

so that the hypotheses of Theorem 1 are satisfied. Hence $(Y, \boldsymbol{R}, g)$ is isomorphic to a radial flow in $l_{2}$. Furthermore, we may assume that the set $S$, in the proof of Theorem 1, is disjoint from $h[X]$. (Otherwise it may be replaced by $\lambda^{-n} S$ with $n$ sufficiently large.) It follows that $\left(X, \boldsymbol{R}^{+}, f\right)$ is isomorphic to a radial semiflow on a subset of the Hilbert Cube.

Proof of Theorem II. As in the preceding proof fix $\lambda, 0<\lambda<1$ and set $\bar{f}(x)=f(x, 1)$. The mapping $\bar{f}: X \rightarrow X$ satisfies the hypo- 
theses of Theorem 2. Hence there is a one-one continuous mapping $h$ of $X$ into the Hilbert Cube in $l_{2}$ such that $h(\bar{f}(x))=\lambda h(x)$ with $h^{-1}$ continuous on $h[X] \backslash\{0\}$.

The rest of the preceding proof applies verbatim with the only change that $(Y, \boldsymbol{R}, g)$ as defined there is homomorphic (rather than isomorphic) to a radial flow on a subset of the Hilbert Cube in $l_{2}$. Hence $\left(X, \boldsymbol{R}^{+}, f\right)$ is homomorphic to such a semiflow.

The author wishes to record his thanks to the Weizmann Institute of Science, Rehovot, Israel and to Michigan State University, East Lansing, Michigan, for the hospitality and assistance extended to him while on sabbatical leave from Dalhousie University, Halifax, N.S.

\section{REFERENCES}

1. M. Edelstein, On the representation of mappings of compact metrizable spaces as restrictions of linear transformations, Canad. J. Math., 22 (1970), 372-375.

2. L. Janos, Linearization of a contractive homeomorphism, Canad. J. Math., 20 (1968), 1387-1390.

3. - A linearization of semiflows in the Hilbert space $l_{2}$, Topology Proceedings, Baton Rouge, Louisiana, 2 (1977) 219-232.

4. V.V. Nemytskii and V.V. Stepanov, Qualitative theory of differential equations (Russian), English translation, Princeton University Press, 1960.

5. K.S. Sybirsky, Introduction to topological dynamics (Russian), English translation, Noordhof Intern. Publ. Leyden, 1975.

Received August 27, 1979.

Dalhousie University

Halifax, N.S. B3H $4 \mathrm{H} 8$

CANADA 



\section{PACIFIC JOURNAL OF MATHEMATICS}

\section{EDITORS}

DONALD BABBITT (Managing Editor)

University of California

Los Angeles, CA 90024

Hugo RossI

University of Utah

Salt Lake City, UT 84112

C. C. MOORE and ANDREW OGG

University of California

Berkeley, CA 94720

\section{J. DugundjI}

Department of Mathematics

University of Southern California

Los Angeles, CA 90007

R. FinN and J. Milgram

Stanford University

Stanford, CA 94305

\section{ASSOCIATE EDITORS}
E. F. BeCKENBACH
B. H. Neumann
F. WoLF
K. YoSHIDA

\section{SUPPORTING INSTITUTIONS}

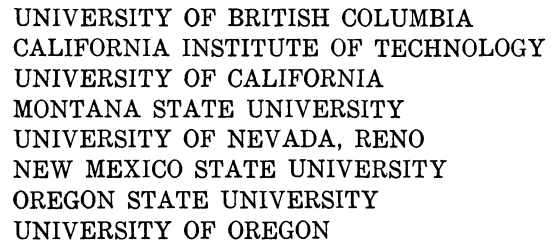

UNIVERSITY OF BRITISH COLUMBIA CALIFORNIA INSTITUTE OF TECHNOLOGY UNIVERSITY OF CALIFORNIA MONTANA STATE UNIVERSITY UNIVERSITY OF NEVADA, RENO NEW MEXICO STATE UNIVERSITY OREGON STATE UNIVERSITY UNIVERSITY OF OREGON

\author{
UNIVERSITY OF SOUTHERN CALIFORNIA \\ STANFORD UNIVERSITY \\ UNIVERSITY OF HAWAII \\ UNIVERSITY OF TOKYO \\ UNIVERSITY OF UTAH \\ WASHINGTON STATE UNIVERSITY \\ UNIVERSITY OF WASHINGTON
}

The Supporting Institutions listed above contribute to the cost of publication of this Journal, but they are not owners or publishers and have no responsibility for its content or policies.

Mathematical papers intended for publication in the Pacific Journal of Mathematics should be in typed form or offset-reproduced, (not dittoed), double spaced with large margins. Please do not use built up fractions in the text of the manuscript. However, you may use them in the displayed equations. Underline Greek letters in red, German in green, and script in blue. The first paragraph or two must be capable of being used separately as a synopsis of the entire paper. Please propose a heading for the odd numbered pages of less than 35 characters. Manuscripts, in triplicate, may be sent to any one of the editors. Please classify according to the scheme of Math. Reviews, Index to Vol. 39. Supply name and address of author to whom proofs should be sent. All other communications should be addressed to the managing editor, or Elaine Barth, University of California, Los Angeles, California, 90024.

50 reprints to each author are provided free for each article, only if page charges have been substantially paid. Additional copies may be obtained at cost in multiples of 50 .

The Pacific Journal of Mathematics is issued monthly as of January 1966. Regular subscription rate: $\$ 84.00$ a year (6 Vols., 12 issues). Special rate: $\$ 42.00$ a year to individual members of supporting institutions.

Subscriptions, orders for numbers issued in the last three calendar years, and changes of address shoud be sent to Pacific Journal of Mathematics, P.O. Box 969, Carmel Valley, CA 93924, U.S.A Old back numbers obtainable from Kraus Periodicals Co., Route 100, Millwood, NY 10546.

PUBLISHED BY PACIFIC JOURNAL OF MATHEMATICS, A NON-PROFIT CORPORATION

Printed at Kokusai Bunken Insatsusha (International Academic Printing Co., Ltd.). 8-8, 3-chome, Takadanobaba, Shinjuku-ku, Tokyo 160, Japan. 


\section{Pacific Journal of Mathematics}

\section{Vol. 91, No. 2 December, 1980}

Victor P. Camillo and Julius Martin Zelmanowitz, Dimension modules ... . . 249

Yonina S. Cooper, Stable sequences in pre-abelian categories ........... 263

Chandrakant Mahadeorao Deo and H. Ship-Fah Wong, On Berry-Esseen approximation and a functional LIL for a class of dependent random fields.........................................

H. P. Dikshit and S. N. Dubey, $|C, 1|$ summability of series associated with

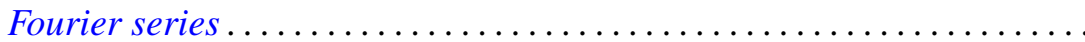

M. Edelstein, On the homomorphic and isomorphic embeddings of a semiflow into a radial flow.

Gilles Godefroy, Compacts de Rosenthal ..................... 293

James Guyker, Commuting hyponormal operators ................ 307

Thomas Eric Hall and Peter R. Jones, On the lattice of varieties of bands of

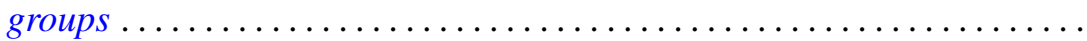

Taqdir Husain and Saleem H. Watson, Topological algebras with orthogonal Schauder bases ....................................

V. K. Jain, Some expansions involving basic hypergeometric functions of two variables. . .

Joe W. Jenkins, On group actions with nonzero fixed points ........... 363

Michael Ellsworth Mays, Groups of square-free order are scarce ........ 373

Michael John McAsey, Canonical models for invariant subspaces... 377

Peter A. McCoy, Singularities of solutions to linear second order elliptic partial differential equations with analytic coefficients by approximation methods...

Terrence Millar, Homogeneous models and decidability.

Stephen Carl Milne, A multiple series transformation of the very well poised

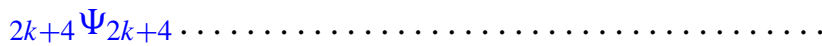

Robert Olin and James E. Thomson, Irreducible operators whose spectra are spectral sets...

Robert John Piacenza, Cohomology of diagrams and equivariant singular

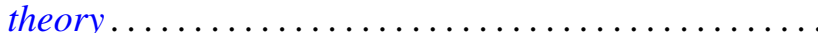

Louis Jackson Ratliff, Jr., Integrally closed ideals and asymptotic prime divisors

Robert Breckenridge Warfield, Jr., Cancellation of modules and groups and stable range of endomorphism rings.................

B. J. Day, Correction to: "Locale geometry" ...............

Stanley Stephen Page, Correction to: "Regular FPF rings" ... 